\title{
Review and Consensus on Pharmacogenomic Testing in Psychiatry
}

Authors

Chad A. Bousman1, 2, 3, 4, Susanne A. Bengesser ${ }^{5}$, Katherine J. Aitchison ${ }^{6}$, Azmeraw T. Amare ${ }^{7,8}$, Harald Aschauer ${ }^{9}$, Bernhard T. Baune ${ }^{10,4}$, 11, Bahareh Behroozi As| ${ }^{6}$, Jeffrey R. Bishop ${ }^{12}$, Margit Burmeister ${ }^{13}$, Boris Chaumette ${ }^{14,}{ }^{15}$, Li-Shiun Chen ${ }^{16}$, Zachary A. Cordner ${ }^{17}$, Jürgen Deckert ${ }^{18}$, Franziska Degenhardt ${ }^{19}$, 20 , Lynn E. DeLisi21, Lasse Folkersen ${ }^{22}$, James L. Kennedy23, 24, Teri E. Klein ${ }^{25}$, Joseph L. McClay ${ }^{26}$, Francis J. McMahon ${ }^{27}$, Richard Musil28, Nancy L. Saccone ${ }^{16}$, Katrin Sangkuhl'25, Robert M. Stowe ${ }^{29}$, Ene-Choo $\operatorname{Tan}^{30}$, Arun K. Tiwari²3, 24, Clement C. Zai ${ }^{23,24}$, Gwyneth Zai ${ }^{23,24}$, Jianping Zhang ${ }^{31}$, Andrea Gaedigk ${ }^{32}$, Daniel J Müller², 24

\section{Affiliations}

1 Departments of Medical Genetics, Psychiatry, Physiology \& Pharmacology, University of Calgary, Calgary, AB, Canada

2 Hotchkiss Brain Institute, Cumming School of Medicine, University of Calgary, Calgary, AB, Canada

3 Alberta Children's Hospital Research Institute, Calgary, AB, Canada

4 Department of Psychiatry, Melbourne Medical School, The University of Melbourne, Melbourne, VIC, Australia

5 Department of Psychiatry and Psychotherapeutic Medicine, Medical University of Graz, Austria

6 Departments of Psychiatry, Medical Genetics and the Neuroscience and Mental Health Institute, University of Alberta, Edmonton, AB, Canada

7 Discipline of Psychiatry, School of Medicine, University of Adelaide, Adelaide, SA, Australia

8 South Australian Health and Medical Research Institute (SAHMRI), Adelaide, SA, Australia

9 Biopsychosocial Corporation (BioPsyC), non-profit association, Vienna, Austria

10 Department of Psychiatry and Psychotherapy, University of Münster, Germany

11 The Florey Institute of Neuroscience and Mental Health, The University of Melbourne, Parkville, VIC, Australia

12 Department of Experimental and Clinical Pharmacology, University of Minnesota College of Pharmacy and Department of Psychiatry, University of Minnesota Medical School, Minneapolis, MN, USA

13 Michigan Neuroscience Institute and Departments of Computational Medicine \& Bioinformatics, Human Genetics and Psychiatry, The University of Michigan, Ann Arbor MI, USA

14 Institute of Psychiatry and Neuroscience of Paris, GHU Paris Psychiatrie \& Neurosciences, University of Paris, Paris, France

15 Department of Psychiatry, McGill University, Montreal, Canada

16 Departments of Psychiatry and Genetics, Washington University School of Medicine in St. Louis, USA

17 Department of Psychiatry \& Behavioral Sciences, Johns Hopkins University School of Medicine, Baltimore, MD, USA
18 Department of Psychiatry, Psychosomatics and Psychotherapy, Center of Mental Health, Würzburg, Germany

19 Institute of Human Genetics, University of Bonn, School of Medicine \& University Hospital Bonn, Bonn, Germany

20 Department of Child and Adolescent Psychiatry, Psychosomatics and Psychotherapy, University Hospital Essen, University of Duisburg-Essen, Duisburg, Germany

21 Department of Psychiatry, Harvard Medical School, Cambridge Health Alliance, Cambridge, Massachusetts, USA

22 Institute of Biological Psychiatry, Capital Region Hospitals, Copenhagen, Denmark

23 Department of Psychiatry, University of Toronto, Toronto, Ontario, Canada

24 Centre for Addiction and Mental Health, University of Toronto, Toronto, Ontario, Canada

25 Department of Biomedical Data Science, Stanford University, Stanford, California, USA

26 Department of Pharmacotherapy and Outcome Science, Virginia Commonwealth University School of Pharmacy, Richmond, VA, USA

27 Human Genetics Branch, National Institute of Mental Health, Bethesda, MD, USA

28 Department of Psychiatry and Psychotherapy, LudwigMaximilians-University, Munich, Germany

29 Departments of Psychiatry and Neurology (Medicine), University of British Columbia, USA

30 KK Research Centre, KK Women's and Children's Hospital, Singapore, Singapore

31 Department of Psychiatry, Weill Cornell Medical College, New York-Presbyterian Westchester Division, White Plains, NY, USA

32 Division of Clinical Pharmacology, Toxicology \& Therapeutic Innovation, Children's Mercy Kansas City, Kansas City and School of Medicine, University of Missouri-Kansas City, Kansas City, MO, USA

Key words precision medicine, pharmacogenetics, prescribing

received $\quad 09.07 .2020$

revised $\quad 05.10 .2020$

accepted $\quad 07.10 .2020$

published online $\quad 04.11 .2020$ 
Bibliography

Pharmacopsychiatry 2021; 54: 5-17

DOI 10.1055/a-1288-1061

ISSN 0176-3679

(c) 2020. Thieme. All rights reserved.

Georg Thieme Verlag KG, Rüdigerstraße 14, 70469 Stuttgart, Germany

\section{Correspondence}

Chad Bousman

Department of Medical Genetics University of Calgary 3330

Hospital Drive NW 228 HMRB T2N 4N1 Calgary

Alberta

Canada

chad.bousman@ucalgary.ca

Daniel Müller

Department of Psychiatry University of Toronto 250 College

Street R132 M5T 1R8 Toronto

Ontario

Canada

Daniel.Mueller@camh.ca

\section{ABSTRACT}

The implementation of pharmacogenomic (PGx) testing in psychiatry remains modest, in part due to divergent perceptions of the quality and completeness of the evidence base and diverse perspectives on the clinical utility of PGx testing among psychiatrists and other healthcare providers. Recognizing the current lack of consensus within the field, the International Society of Psychiatric Genetics assembled a group of experts to conduct a narrative synthesis of the PGx literature, prescribing guidelines, and product labels related to psychotropic medications as well as the key considerations and limitations related to the use of PGx testing in psychiatry. The group concluded that to inform medication selection and dosing of several commonly-used antidepressant and antipsychotic medications, current published evidence, prescribing guidelines, and product labels support the use of PGx testing for 2 cytochrome P450 genes (CYP2D6, CYP2 (19). In addition, the evidence supports testing for human leukocyte antigen genes when using the mood stabilizers carbamazepine (HLA-A and HLA-B), oxcarbazepine (HLA-B), and phenytoin (CYP2C9, HLA-B). For valproate, screening for variants in certain genes ( $P O L G, O T C, C S P 1)$ is recommended when a mitochondrial disorder or a urea cycle disorder is suspected. Although barriers to implementing PGx testing remain to be fully resolved, the current trajectory of discovery and innovation in the field suggests these barriers will be overcome and testing will become an important tool in psychiatry.

\section{Introduction}

The treatment of psychiatric disorders commonly involves the use of psychotropic medications such as antidepressants, antipsychotics, mood stabilizers, anxiolytics/hypnotics, stimulants, or anti-addiction medications. However, recipients of these medications often experience a lengthy trial-and-error process marked by poorly managed symptoms and/or adverse drug reactions before the right medications and doses are established. As such, strategies to predict or mitigate these poor responses are needed. Current pharmacological strategies include scheduled titrations over time (sometimes guided by therapeutic drug monitoring) [1] until a patient receives a standard target dose thought to be sufficient for clinical efficacy. Yet the same dose may not be the correct one for all individuals. Another emerging and complementary strategy is the implementation of pharmacogenomic (PGx) testing to inform medication selection and dosing decisions [2]. PGx testing examines genetic variation involved in medication metabolism and action to facilitate individualized prescribing, thus reducing undesirable outcomes. To date, this strategy has been implemented in a growing number of medical centres around the world and has fueled a burgeoning commercial PGx testing sector [3-5]. However, widespread implementation and adoption of this strategy has not yet occurred in psychiatry, in part due to diverging perceptions of the quality and completeness of the PGx evidence base, variable knowledge among psychiatrists about genetics, and mixed views related to the utility of $P G x$ testing in clinical practice. Recognizing the current lack of consensus within the field, the International So- ciety of Psychiatric Genetics (ISPG) assembled a group of experts to provide an overview of PGx mechanisms, summarize the current evidence and treatment recommendations related to PGx in psychiatry, and provide consensus recommendations for the use of PGx testing in clinical practice [6]. This review discusses the evidence that was considered by the ISPG and provides an up-to-date summary of recent developments that clinicians should know when considering PGx testing for their patients.

\section{Pharmacogenomic Mechanisms}

\section{Pharmacokinetics}

The majority of medications used to treat psychiatric conditions undergo hepatic metabolism, although some, such as lithium, are eliminated only through the kidneys. A number of genes encoding oxidative (Phase 1 ) and conjugative (Phase 2 ) metabolizing enzymes contain variants known to influence enzymatic activity. In addition, genetic variation in drug transporters expressed in the liver, gut, and at the blood brain barrier may alter the distribution of drugs and thereby alter their pharmacokinetic profile. The drug metabolizing enzymes that are currently the most clinically relevant to commonly used psychiatric medications are the cytochrome P450 (CYP) enzymes CYP2C9, CYP2C19, and CYP2D6 [7]. While genes encoding conjugative enzymes, such as UDP-glucuronosyltransferase (UGT) and catechol-O-methyltransferase $(C O M T)$ enzymes along with the P-glycoprotein ( $A B C B 1)$ drug trans- 
porter, may also be relevant, their clinical utility has not yet been established.

The CYP superfamily is arguably the most important enzyme system for drug metabolism. Allelic variants of CYP genes are commonly referred to using the $\operatorname{star}\left({ }^{*}\right)$ nomenclature $[8,9]$. Genotypes (reported as star diplotypes, e.g., CYP2D6 * $1 /{ }^{*} 2$ ) are then translated into metabolizer phenotypes. The most widely used phenotype classification system includes: ultrarapid metabolizers (UMs), rapid metabolizers (RMs), normal metabolizers (NMs, activity of reference or sum of allelic variants with activity that is similar to that of the reference), intermediate metabolizers (IMs), and poor metabolizers (PMs, little or no enzyme activity) [10]. In this context, "activity" refers to the metabolic capacity of an enzyme, which broadly includes catalytic activity and enzyme abundance [10].

\section{Pharmacodynamics}

Pharmacodynamics refers to the biochemical, cellular, and physiologic effects of medications and their mechanism of action [11]. In psychiatric PGx, the focus has historically been on variation in genes encoding neurotransmitter receptors and reuptake transporters that are located on the pre- or postsynaptic cell membranes. More recently, the focus has expanded to include genes involved in signal transduction, gene transcription, and protein folding and trafficking. However, our understanding of how genetic variation affects the pharmacodynamics of psychiatric medications is still evolving.

\section{Immunologic mechanisms}

Immunologic mechanisms are often involved in drug hypersensitivity reactions. Variations in some human leukocyte antigen (HLA) genes are implicated in the risk for potentially severe and fatal hypersensitivity reactions to certain anticonvulsants/mood stabilizers [12]. More details are provided below in the section related to mood stabilizers.

\section{Pharmacogenomic Evidence and Guidelines for Psychiatry}

\section{Antidepressants}

\section{Evidence}

The bulk of antidepressant PGx evidence has been derived from studies on major depressive disorder and has focused on pharmacokinetic mechanisms, which have been reviewed in detail elsewhere [13]. In brief, findings have shown that genetic variants in CYP2C19 and CYP2D6 are associated with antidepressant blood concentrations, adverse drug reactions, and, to a lesser extent, clinical outcomes such as treatment discontinuation or symptom response $[14,15]$. From a pharmacodynamic perspective, the Sequenced Treatment Alternatives to Relieve Depression (STAR * D) study [16], the Genome-based Therapeutic Drugs for Depression (GENDEP) project $[17,18]$, and the Munich Antidepressant Response Signature (MARS) [19], as well as the International SSRI Pharmacogenomics Consortium GWAS analysis [20], have not consistently supported any single pharmacodynamic gene variant as a significant predictor of antidepressant treatment response. The Pharma- cogenomics Knowledgebase (PharmGKB) contains clinical annotations summarizing literature findings for associations between antidepressant efficacy and potentially relevant genes such as SLC6A4 (serotonin transporter), HTR2A (serotonin 2A receptor), GRIK4 (glutamate ionotropic receptor kainate 4), and FKBP5 (FK506 binding protein 5). However, the associations have only moderate or low levels of evidence [21].

\section{Guidelines}

There is disagreement about the role of PGx testing in antidepressant prescribing. A recent safety communication from the US Food and Drug Administration (FDA) cautioned against using PGx testing to guide antidepressant prescribing, citing lack of evidence [22]. However, as clarified by us previously [23], 17 antidepressants have been included in published PGx-based prescribing guidelines $[13,24]$ or product labels for associations with CYP2C19 and/or CYP2D6 ( Table 1). The Clinical Pharmacogenetic Implementation Consortium (CPIC) guidelines for CYP2C19 PMs suggest a $50 \%$ reduction of the recommended starting dose of citalopram, escitalopram, sertraline, and tertiary amine tricyclic antidepressants (e. g., amitriptyline); whereas RMs/UMs treated with citalopram, escitalopram, and tertiary amine tricyclic antidepressants would likely have inadequate treatment response due to inadequate circulating antidepressant blood levels and thus may benefit from an alternative antidepressant $[13,24]$. For CYP2D6 PMs, CPIC recommends up to a $50 \%$ reduction of most tricyclic antidepressants, fluvoxamine, and paroxetine doses, while for UMs, it is advised to select an alternative antidepressant that is not predominantly metabolized by CYP2D613, 24]. In addition, the Dutch Pharmacogenetics Working Group (DPWG) recommends reduced dosing (amount unspecified) of venlafaxine for CYP2D6 PMs and up to $150 \%$ increased dosing for UMs $[25,26]$.

\section{Antipsychotics}

\section{Evidence}

Most antipsychotics are hepatically metabolized by one or more CYP450 enzymes. To date, studies of pharmacokinetic genes have predominantly focused on CYP2D6 genetic variation, with risperidone and aripiprazole receiving the most recent attention [27-29]. In contrast, evidence relating to the impact of pharmacodynamic genes on antipsychotics drug response is still emerging. It is well established that antipsychotics act primarily via antagonism [30] or partial-antagonism [31] of the dopamine $D_{2}$ receptor. However, evidence linking genetic variation in the dopamine $D_{2}$ receptor (DRD2) gene to antipsychotic efficacy or adverse reactions has been inconsistent [32].

\section{Guidelines}

To date, 10 antipsychotics have product labels or prescribing guidelines [25] that offer selection or dosing recommendations based on CYP2D6 metabolizer status ( $\triangleright$ Table 2). For all of these drugs, the guidelines or product labels recommend that CYP2D6 PMs receive lower starting doses or an alternative drug not primarily metabolized by CYP2D6. In addition, the DPWG guidelines recommend reductions in the starting dose for pimozide and zuclopenthixol among CYP2D6 IMs, while for UMs they recommend the use of an alternative drug or titration to the maximum dose for haloperidol, 
- Table 1 Actionable pharmacogenetic guidelines and product labels by antidepressants.

\begin{tabular}{|c|c|c|c|c|c|c|}
\hline \multirow[b]{2}{*}{ Antidepressant } & \multicolumn{2}{|c|}{ Actionable Guideline Available ${ }^{1}$} & \multicolumn{4}{|l|}{ Product Label $^{2}$} \\
\hline & CPIC & DPWG & FDA & EMA & PMDA & HCSC \\
\hline Amitriptyline & CYP2C19, CYP2D6 & CYP2D6 & CYP2D6 & - & - & - \\
\hline Amoxapine & - & - & CYP2D6 & - & - & - \\
\hline Citalopram & CYP2C19 & CYP2C19 & CYP2C19 & - & - & CYP2C19 \\
\hline Clomipramine & CYP2C19, CYP2D6 & CYP2D6 & CYP2D6 & - & - & - \\
\hline Desipramine & CYP2D6 & - & CYP2D6 & - & - & - \\
\hline Doxepin & CYP2C19, CYP2D6 & CYP2D6 & CYP2C19, CYP2D6 & - & - & - \\
\hline Duloxetine & - & - & CYP2D6 & CYP2D6 & - & - \\
\hline Escitalopram & CYP2C19 & CYP2C19 & - & - & CYP2C19 & - \\
\hline Fluvoxamine & CYP2D6 & - & CYP2D6 & - & - & - \\
\hline Imipramine & CYP2C19, CYP2D6 & CYP2C19, CYP2D6 & CYP2D6 & - & - & - \\
\hline Nortriptyline & CYP2D6 & CYP2D6 & CYP2D6 & - & - & CYP2D6 \\
\hline Paroxetine & CYP2D6 & CYP2D6 & - & - & - & - \\
\hline Protriptyline & - & - & CYP2D6 & - & - & - \\
\hline Sertraline & CYP2C19 & CYP2C19 & - & - & - & - \\
\hline Trimipramine & CYP2C19, CYP2D6 & - & CYP2D6 & - & - & - \\
\hline Venlafaxine & - & CYP2D6 & CYP2D6 & - & - & - \\
\hline Vortioxetine & - & - & CYP2D6 & CYP2D6 & - & CYP2D6 \\
\hline \multicolumn{7}{|c|}{$\begin{array}{l}\text { CPIC: Clinical Pharmacogenetics Implementation Consortium; DPWG: Dutch Pharmacogenetics Working Group; EMA: European Medicines Agency; } \\
\text { FDA: US Food and Drug Administration; HCSC: Health Canada (Santé Canada); PMDA: Pharmaceuticals and Medical Devices Agency, Japan. 'Only } \\
\text { guidelines where a clinical action has been recommended were included. Product label information was extracted from the Pharmacogenomics } \\
\text { Knowledgebase (PharmGKB), only labels coded as "actionable," "test recommended," or "test required” by PharmGKB curators were included. For a } \\
\text { description of these categories (PGx levels) and the drug label curation process, see https://www.pharmgkb.org/page/drugLabelLegend. Drugs } \\
\text { reviewed that did not have an actionable guideline or product label included: agomelatine, buproprion, desvenlafaxine, fluoxetine, levomilnacipran, } \\
\text { mianserin, mirtazapine, milnacipran, nefazodone, phenelzine, reboxetine, selegiline, tranylcypromine, trazodone, and vilazodone. }\end{array}$} \\
\hline
\end{tabular}

- Table 2 Actionable pharmacogenetic guidelines and product labels by antipsychotics.

\begin{tabular}{|c|c|c|c|c|c|c|}
\hline \multirow[b]{2}{*}{ Antipsychotic } & \multicolumn{2}{|c|}{ Actionable Guideline Available ${ }^{1}$} & \multicolumn{4}{|c|}{ Product Label $^{2}$} \\
\hline & CPIC & DPWG & FDA & EMA & PMDA & HCSC \\
\hline Aripiprazole & - & CYP2D6 & CYP2D6 & CYP2D6 & - & CYP2D6 \\
\hline Brexpiprazole & - & CYP2D6 & CYP2D6 & CYP2D6 & - & - \\
\hline Clozapine & - & - & CYP2D6 & - & - & - \\
\hline Haloperidol & - & CYP2D6 & - & - & - & - \\
\hline Iloperidone & - & - & CYP2D6 & - & - & - \\
\hline Perphenazine & - & - & CYP2D6 & - & CYP2D6 & - \\
\hline Pimozide & - & CYP2D6 & CYP2D6 & - & - & - \\
\hline Risperidone & - & CYP2D6 & - & - & - & - \\
\hline Thioridazine & - & - & CYP2D6 & - & - & - \\
\hline Zuclopenthixol & - & CYP2D6 & - & - & - & - \\
\hline \multicolumn{7}{|c|}{$\begin{array}{l}\text { CPIC: Clinical Pharmacogenetics Implementation Consortium; DPWG: Dutch Pharmacogenetics Working Group; EMA: European Medicines Agency; } \\
\text { FDA: US Food and Drug Administration; HCSC: Health Canada (Santé Canada); PMDA: Pharmaceuticals and Medical Devices Agency, Japan. 'Only } \\
\text { guidelines where a clinical action has been recommended were included. 2Product label information was extracted from the Pharmacogenomics } \\
\text { Knowledgebase (PharmGKB), only labels coded as "actionable," "test recommended," or "test required" by PharmGKB curators were included. For a } \\
\text { description of these categories (PGx levels) and the drug label curation process, see https://www.pharmgkb.org/page/drugLabelLegend. Drugs } \\
\text { reviewed that did not have an actionable guideline or product label included: asenapine, cariprazine, chlorpromazine, fluphenazine, loxapine, } \\
\text { lurasidone, olanzapine, paliperidone, promethazine, quetiapine, thiothixene, trifluoperazine, and ziprasidone. }\end{array}$} \\
\hline
\end{tabular}

risperidone, and zuclopenthixol. Of note, the FDA product label for clozapine suggest CYP2D6 PMs may require a dose reduction, despite CYP2D6's minor role (6\%) in the metabolism of clozapine [33], and a recent study that showed CYP2D6 genotype-predicted enzyme activity explained a minimal amount of the variance (3\%-7\%) in dose-adjusted clozapine levels and psychotic symptom severity [34]. In addition, the FDA product label for pimozide states CYP2D6 genetic testing should be performed if doses above 0.05 
> Table 3 Actionable pharmacogenetic guidelines and product labels by mood stabilizers/anticonvulsants.

\begin{tabular}{|c|c|c|c|c|c|c|}
\hline \multirow[b]{2}{*}{ Mood stabilizers/ anticonvulsants } & \multicolumn{2}{|c|}{${\text { Actionable Guideline Available }{ }^{1}}$} & \multicolumn{4}{|l|}{ Product Label $^{2}$} \\
\hline & CPIC & DPWG & FDA & EMA & PMDA & HCSC \\
\hline Carbamazepine & HLA-A, HLA-B & - & HLA-A, HLA-B & - & HLA-A, HLA-B & HLA-A, HLA-B \\
\hline Oxcarbazepine & HLA-B & - & HLA-B & - & - & HLA-B \\
\hline Phenytoin & CYP2C9, HLA-B & CYP2C9 & HLA-B & - & - & HLA-B \\
\hline Valproic acid & - & - & OTC, POLG & - & CPS1, OTC & OTC, POLG \\
\hline \multicolumn{7}{|c|}{$\begin{array}{l}\text { CPIC: Clinical Pharmacogenetics Implementation Consortium; DPWG: Dutch Pharmacogenetics Working Group; EMA: European Medicines Agency; } \\
\text { FDA: US Food and Drug Administration; HCSC: Health Canada (Santé Canada); PMDA: Pharmaceuticals and Medical Devices Agency, Japan. 'Only } \\
\text { guidelines where a clinical action has been recommended were included. Product label information was extracted from the Pharmacogenomics } \\
\text { Knowledgebase (PharmGKB), only labels coded as "actionable," "test recommended," or "test required" by PharmGKB curators were included. For a } \\
\text { description of these categories (PGx levels) and the drug label curation process, see https://www.pharmgkb.org/page/drugLabelLegend. Drugs } \\
\text { reviewed that did not have an actionable guideline or product label included: eslicarbazepine, gabapentin, lamotrigine, levetiracetam, lithium, } \\
\text { phenobarbital, pregabalin, topiramate, vigabatrin, and zonisamide. }\end{array}$} \\
\hline
\end{tabular}

$\mathrm{mg} / \mathrm{kg} /$ day in children or above $4 \mathrm{mg} /$ day in adults will be used. However, other regulatory agencies seem not to mention testing for CYP2D6 on their pimozide labels ( $\bullet$ Table 2 ).

\section{Mood stabilizers/anticonvulsants}

\section{Evidence}

In contrast to antidepressants and antipsychotics, there is limited evidence supporting a link between genetic variation in pharmacokinetic genes and mood stabilizer/anticonvulsant treatment outcomes. An exception is the strong associations between CYP2C9 IMs and PMs and increased phenytoin plasma concentrations [35]. Furthermore, there are no robust associations between pharmacodynamic gene variants and mood stabilizer/anticonvulsant treatment outcomes. Three independent GWASs have identified SNPs associated with lithium response, but each study implicates a different locus [36-38]. Polygenic risk scores derived from schizophrenia and depression GWAS have been associated with lithium response $[39,40]$, but none of these findings have been replicated.

The immunologic genes HLA-A and HLA-B are robustly linked to rare, but potentially fatal, severe cutaneous adverse reactions (SCARs) (e. g., Stevens-Johnson syndrome [SJS] and toxic epidermal necrolysis [TEN]) following exposure to carbamazepine, oxcarbazepine, and phenytoin [41]. Specifically, $H L A-A{ }^{*}$ 31:01 and $H L A-B{ }^{*} 15: 02$ alleles are associated with a higher risk of SCARs if exposed to carbamazepine [12], while only the HLA-B ${ }^{*} 1502$ allele is linked to a higher risk of SCARs following exposure to oxcarbazepine and phenytoin [42]. Notably, a recent meta-analysis of 11 studies in Asian (Chinese, Korean, and Thai) populations found a pooled odds ratio of 2.4 for risk of lamotrigineinduced SJS/TEN in HLA-B ${ }^{*}$ 15:02 carriers [43].

\section{Guidelines}

Product labels and prescribing guidelines are available for carbamazepine, oxcarbazepine, and phenytoin ( $\vee$ Table 3 ). For carbamazepine and oxcarbazepine, the FDA-approved labels recommend testing for the $H L A-B{ }^{*} 1502$ allele prior to prescribing these medications to "genetically at-risk populations." Current evidence suggests at-risk individuals are those of Han Chinese, Thai, Vietnamese, Indonesian, Malay, Filipino, or Indian descent, who carry this allele more frequently (3-36\%) [44, 45]. In fact, in Taiwan [46], Hong Kong [47], and Thailand [48], HLA testing prior to prescrib- ing carbamazepine and oxcarbazepine is standard practice. The FDA-approved label for carbamazepine also provides information about HLA-A * 31:01 and HLA-B ${ }^{*} 1502$. Other regulatory agencies such as Health Canada (HCSC) and the Pharmaceuticals and Medical Devices Agency (PMDA) in Japan also note the risk of prescribing carbamazepine to individuals that carry the HLA-A *31:01 or $H L A-B * 1502$ alleles. Aligned with these product label recommendations, CPIC recommends use of alternative medications for individuals who test positive for HLA-A * 31:01 (carbamazepine) or HLA$B^{*} 1502$ (carbamazepine, oxcarbazepine, and phenytoin). Furthermore, the CPIC guideline for phenytoin recommends a $50 \%$ dose reduction for CYP2C9 PMs, assuming the individual is not a carrier of the HLA-B ${ }^{*} 1502$ allele $[35,44]$.

Finally, FDA, HCSC, and the PMDA product labels include language that valproic acid is contraindicated or recommend genetic tests before prescribing valproic acid to individuals suspected (e. g., by family history) of having certain rare metabolic disorders. Sequencing of the gene POLG (mitochondrial DNA polymerase $\gamma$ ) is recommended in patients suspected of having a mitochondrial disorder, while patients suspected of having a urea cycle disorder should be screened for mutations in the genes OTC (ornithine transcarbamylase) and CPS1 (carbamoyl-phosphate synthase 1). Use of valproic acid by these individuals can induce liver toxicity, hyperammonemia, and encephalopathy [49].

\section{Anxiolytics/hypnotics}

\section{Evidence}

Most anxiolytic/hypnotic medications are preferentially metabolized by CYP3A4, CYP3A5, and CYP2C19[21]. Links between anxiolytic/hypnotic treatment outcomes and CYP3A4 or CYP3A5 genetic variation have been inconsistent [21], while associations between CYP2C19 allelic variation and anxiolytic/hypnotic concentrations are more robust. This is particularly the case for clobazam and, to a lesser extent, diazepam. Serum concentrations of clobazam were increased $30-50 \%$ and norclobazam (active metabolite) concentrations were up to 7 -fold higher in CYP2C19 PMs relative to other metabolizer groups [50], with single and repeated dosing half-lives in PMs of 130 hours and 289 hours, respectively [51]. Likewise, for diazepam and its active metabolite (nordiazepam), CYP2C19 PMs had $40 \%$ and $75 \%$ higher plasma half-lives compared to NMs, re- 
spectively [52]. There is also some evidence linking the UGT2B15 (UDP-glucuronosyltransferase 2B15) rs1902023:AA genotype with reduced clearance of lorazepam and oxazepam [21]. In contrast, the limited available data do not suggest that any pharmacodynamic gene or variant is robustly associated with response to anxiolytic/hypnotic medications [53].

\section{Guidelines}

There are 2 actionable gene-drug pairs included on FDA-approved product labels, CYP2C19 and clobazam and CYP2C19 and diazepam. For clobazam, the FDA recommends that CYP2C19 PMs receive a starting dose of $5 \mathrm{mg} /$ day, with up-titrations proceeding slowly according to body weight. For diazepam, the label does not provide specific dosing recommendations but does note that CYP2C19PMs could present with marked differences in drug clearance, suggesting caution and additional monitoring is warranted when prescribing this drug to CYP2C19PMs.

\section{ADHD medications}

\section{Evidence}

Stimulants, including methylphenidate and amphetamine, and the nonstimulant atomoxetine, are generally the first line treatments to alleviate core ADHD symptoms. To date, the strongest evidence for the impact of CYP2D6 genotype on atomoxetine has come from pharmacokinetic studies and clinical outcomes in large fixed-dose treatment trials. This body of work, recently reviewed by CPIC and summarized in their consensus guideline [54], demonstrates that using standard dosing approaches, non-PMs are less likely than PMs to achieve blood concentrations (> 200ng/ml) necessary for clinical effectiveness. In contrast, PMs are more likely to experience improvement in ADHD symptoms, but due to their absence of CYP2D6 metabolic activity, they are at also at increased risk of having side effects from atomoxetine and may therefore require lower doses. From a pharmacodynamic perspective, there are a number of interesting findings warranting further investigation related to dopamine and norepinephrine disposition in the brain (e.g., COMT), as well as the contribution of genetic variability in CES1 (carboxylesterase 1) to methylphenidate metabolism [55]. However, the clinical efficacy and utility of testing for these genes remains unknown.

\section{Guidelines}

At the present time, only CYP2D6 is noted as a PGx biomarker that may be helpful in guiding treatment with atomoxetine. Official FDA product labeling, CPIC [54], and DPWG [25] all note the clinical relevance of CYP2D6 genetic variation for atomoxetine prescribing. In the product labeling, patients taking a CYP2D6 inhibitor or who are known CYP2D6 PMs are recommended to start at the same dose as NMs, but to approach dose escalation differently by only considering increases after 4 weeks if the drug is tolerated and symptoms do not improve. CPIC guidelines offer more specific recommendations with respect to CYP2D6 genotype-informed therapy (i. e., specific starting doses, titration, and drug exposure/plasma verification recommendations for children and adults) [54].

\section{Addiction medications}

Evidence

Among substance use disorders and behaviors, several pharmacokinetic and pharmacodynamic genes have been studied, some of which are promising. Markers in the nicotine-metabolizing gene
CYP2A6 have repeatedly been associated with cessation treatment success [56-58] and a randomized, double-blind placebo-controlled trial suggested that CYP2A6 genotype-guided therapy could help improve outcomes for various smoking cessation interventions [59]. Likewise, for CYP2B6, particularly the ${ }^{*} 6$ decreased function allele, has repeatedly been associated with higher methadone plasma concentrations [60], but the magnitude of this effect casts doubt upon the suitability of this marker for use in the clinic [61].

Beyond pharmacokinetics, a number of GWAS have identified candidate variants for tobacco, alcohol, and opioid use behaviors $[62,63]$, although replication of these findings is still required. However, recent work has demonstrated that variation in the $\alpha 5$ nicotinic cholinergic receptor (CHRNA5) gene has prognostic significance for smoking cessation and response to nicotine replacement therapy [56-58]. Specifically, individuals with CHRNA5 genetic variants that increase the risk for heavy smoking and tobacco use disorder are also more likely to benefit from pharmacotherapy for smoking cessation, compared to those who lack the risk variants. In people with alcohol dependence, a variant of the mu opioid receptor gene (OPRM1), rs1799971 (A118G), has been repeatedly associated with reduced analgesic response to exogenous opioids as well as reduced relapse rates during naltrexone treatment [61]. However, a large meta-analysis study has indicated that the effect of the $\mathrm{A} 118 \mathrm{G}$ variant on substance dependence per se is only modest [64].

\section{Guidelines}

At the time of this review, there were no PGx guidelines or product labels for addiction medications due to the relatively limited evidence base.

\section{Pharmacogenomic Testing in Psychiatry}

The PGx evidence to date suggests genetic variation in CYP2D6, CYP2C19, CYP2C9, HLA-A, and HLA-B should be considered when prescribing several medications used in psychiatry. However, to facilitate the implementation of PGx into clinical practice, the mechanisms for testing, reporting, and interpreting the genomic variations associated with the tested genes, as well as understanding the complexities and limitations of testing, are required [65]. In this section, we provide an overview of PGx testing as it relates to psychiatry and highlight some of the challenges and limitations one should consider when using PGx in clinical practice.

\section{Test providers}

PGx test providers are typically classified into 2 groups: commercial and non-commercial. The number of test providers in each of these groups is difficult to estimate. Recent estimates suggest there are over 75 laboratories in the US that offer PGx testing [3]. In addition, many laboratories participate in the Genetic Testing Registry that is maintained by the National Center for Biotechnology Information (https://www.ncbi.nlm.nih.gov/gtr/), and CPIC lists a growing number of clinics, medical centers, and healthcare organizations/systems around the world that have implemented PGx into clinical practice.

The 2 most frequently used implementation models by commercial providers are the gatekeeper and the direct-to-consumer 
(DTC) models [66]. The major difference between these 2 models is the degree to which a healthcare provider's involvement is required to order and/or interpret test results. Within the gatekeeper model, a healthcare provider must be involved in the ordering and interpretation, or in some cases only the ordering or only the interpretation of the test. In contrast, the DTC model does not require the involvement of a healthcare provider in the ordering or interpretation process, although some DTC companies offer consultation/interpretation services with an in-house pharmacist or physician. Non-commercial PGx test providers (i. e., healthcare organizations/systems) typically restrict testing to their specific patient population and require ordering and interpretation of test results by a healthcare provider. However, delivery of the test results varies by non-commercial providers due to differences in the clinical workflows, reimbursement environment, and information technology resources available [66].

\section{Test content}

PGx tests may include a single gene or a panel of genes, although multiple-gene panels have become the norm [3,4]. Evaluations of commercial PGx testing panels have shown that gene content varies from test-to-test and often includes genes lacking sufficient evidence to guide prescribing in psychiatry (e. g., COMT, CYP1A2, $D R D 2, S L C 6 A 4)[4,67,68]$. Thus, the number of genes included on a testing panel is not an adequate metric for test selection. In psychiatry, the gene content most relevant to clinical practice, as discussed in the preceding sections, includes CYP2D6, CYP2C19, CYP2C9, HLA-A, and HLA-B B[65] and most commercial and noncommercial providers test for CYP2D6, CYP2C19, and CYP2C9 [67]. However, even when the same genes appear on a testing panel, the number of sequence variations, or alleles, assayed within those genes can substantially vary among tests [68]. Unfortunately, regulatory standards for PGx test content have not been established. The FDA has recently issued warnings related to PGx testing that has specifically questioned the testing of particular gene-drug pairs to inform prescribing of psychiatric medications [22], which has commenced a discussion on FDA's role in the regulation of PGx testing [23] and has raised concerns related to the content validity and potential detrimental impact of PGx testing panels that include genes with limited supporting evidence [69]. However, the Association for Molecular Pathology (AMP) and College of American Pathologists (CAP) have published recommendations for clinical genotyping allele selection for CYP2C9 [70] and CYP2C19 [71] with a CYP2D6 allele selection guide underway. To enable full use of these guides, test providers should be transparent about which SNPs are tested and not just provide genotype calls or genotype-derived phenotype assignments. A decision tree for guiding test selection is provided elsewhere [72].

\section{Test analytical validity}

PGx testing is ideally performed in laboratories that have been evaluated and accredited according to national regulatory standards to ensure a high level of analytical validity (i.e., ability of a test to detect whether a specific genetic variant is present or absent). However, analytical validity does vary among accredited laboratories. This variability stems from challenges in accurately calling "star" alleles (or haplotypes) from the variants tested, identifica- tion of structural variation (e. g., gene copy number variants, or CNVs), and the presence of novel or rare allelic variants that might affect PCR-based amplification and subsequent genotyping/sequencing. Genotyping technologies are less uniform in the detection of structural variants than in the detection of SNPs or short insertion/deletion polymorphisms. For example, many tests that detect CYP2D6 CNVs often only report the presence of a "duplication" without specifying which allele is "duplicated" and default the copy number to " 2 " without determining how many copies of the gene are actually present. This can lead to inaccurate phenotype assignments, which in turn may lead to inaccurate recommendations. There are also numerous so-called hybrid genes that are part CYP2D6 and part CYP2D7 and do not usually encode a functional enzyme. Detailed descriptions of these structural variants and their impact for psychiatry are described elsewhere [73, 74].

Another challenge for PGx testing is the detection of rare variants. Current PGx testing panels do not typically include rare variants and are also not designed to detect novel variants. Sequencing has the advantage of detecting rare variants that are not part of $P G x$ panels. It has been estimated that rare variants may account for up to $20-30 \%$ of the variance in interindividual response to medications [75]. However, it needs to be emphasized that the functional impact of a rare or novel allele may be uncertain or unknown, and thus clinical interpretation of genotypes containing such variants is often difficult.

\section{Test feasibility}

The feasibility of PGx testing can be a challenge in clinical settings and is dependent on 1) availability of testing, 2) patient and provider acceptability of testing, 3) testing turnaround times, and 4) testing affordability. The exponential growth of PGx testing over the last decade, particularly in the US, has resulted in an increase in testing availability. Likewise, providers and the general public report positive opinions related to PGx testing [76-80], and patient's perception of care improves when testing is delivered [81]. However, strategies for reducing turnaround times and the monetary costs of performing PGx testing are still evolving. Turnaround times range from 1 day to 3 weeks [4], which can reduce the practicality of testing particularly in acute care settings, where expedited prescribing decisions are required. This situation will improve as rapid testing technologies delivering results within an hour emerge $[82,83]$. From a cost perspective, $P G x$ testing remains unattainable for many due to the high out-of-pocket expense and limited third-party reimbursement, although several third-party payers have recently announced limited coverage of testing or are actively evaluating the value of offering such coverage [84].

\section{Test clinical efficacy and cost-effectiveness}

Establishing clinical efficacy and cost-effectiveness of PGx testing is vital to widespread clinical uptake and adoption. Two meta-analytic evaluations of the clinical efficacy of commercial PGx testing in psychiatry have been conducted for prospective and retrospective clinical trials and showed that testing improves the likelihood of achieving symptom remission compared to treatment as usual $[85,86]$. However, recent inconclusive or negative trial findings have been reported $[87,88]$, leading some to conclude that commercial PGx testing is not ready for widespread use in psychiatry 
[89]. Furthermore, evidence of clinical efficacy has primarily been constrained to adults of European-ancestry with major depressive disorder who had a history of antidepressant non-response or adverse drug reactions, suggesting evaluations of clinical efficacy in other clinical populations (e. g., non-Europeans, treatment-naïve, children, schizophrenia) are required.

The cost-effectiveness of PGx testing has been evaluated in retrospective [90,91] and prospective clinical trials [92-94] for both psychotropic and non-psychotropic drugs in diverse clinical settings. The majority of these evaluations have concluded that PGx testing is a cost-effective or cost-saving strategy relative to treatment as usual [90, 95], although limitations have been noted [92], and most economic studies have been completed by providers of commercial PGx testing. Nevertheless, findings to date are aligned with the notion that tailoring drug therapy to an individual's PGx profile can reduce visits to healthcare providers and pharmacy costs related to medication switching as well as emergency room visits and hospitalizations due to adverse drug reactions.

\section{Test results interpretation and delivery}

For most psychiatrists and other healthcare professionals, the interpretation of PGx test results can be a challenge without accompanying clinical decision support. Clinical decision support can be provided in a variety of forms, most commonly through interpretative clinical reports that translate raw PGx data into clinical recommendations and in ideal cases interruptive alerts implemented within the electronic medical record.

The translation process, however, is not trivial. The process includes assigning a function to the alleles possessed by an individual and then combining those functions to derive a phenotype. For some genes, such as CYP2D6, recommendations have been published with the goal to standardize the genotype to phenotype translation [96]. However, this process remains inconsistent across test providers and no gold standard approach exists. Some providers combine information from several genes (combinatorial approach) and employ proprietary algorithms that utilize-to varying degrees-the published literature, product labels, and/or guidelines developed by expert groups to derive recommendations [97]. This variability in genotype to phenotype translation and clinical decision support from one test provider to another can lead to potential discordant recommendations [98]. In addition, third-party analytic applications are now ubiquitously available and are capable of analyzing the raw data available from DTC providers, although the validity of the results produced by these applications have been questioned [99].

Beyond PGx information, other factors such as age [100], sex [101], concomitant medications [102], renal/hepatic function [103], inflammation [104, 105], lifestyle (e. g., smoking, diet), and weight [106] are also important considerations when applying PGx test results (see [107] for a detailed review of these factors). However, most PGx test providers do not typically account for these factors in their clinical decision support, and as such, it is the responsibility of the healthcare provider to be aware and understand how these factors may influence the PGx-based recommendations being offered. For example, an individual genotyped as a NM for a CYP enzyme who is taking a strong inhibitor of that enzyme will phenotypically resemble a PM, while a UM may convert to an IM. Weak inhibitors may convert a NM to an IM and a UM to a NM. This phenomenon is known as phenocopying. Likewise, an individual genotyped as a NM for a CYP enzyme who is taking a potent inducer of that enzyme will phenotypically resemble an UM. In these clinical scenarios, recommendations provided by a typical PGx test report, which does not account for the presence of concomitant inhibitors or inducers, could be misleading or lead to inappropriate medication selection or dosing. When possible, the use of therapeutic drug monitoring in conjunction with PGx testing in these scenarios can confirm suspected phenocopying and ensure more appropriate medication selection or dosing $[108,109]$.

Finally, ancestry is an important factor to consider when interpreting PGx results. There are marked differences in allele frequencies across ancestry groups for most of the genes of key drug metabolizing enzymes. In addition, there are also many non-functional alleles that are relatively rare and have been found in only some populations but not in others [110], resulting in notable differences in phenotype frequencies ( $>$ Table 4) [21]. This makes it particularly challenging to design "one-size-fits-all” test panels, and in practice, most panels are biased toward alleles observed in individuals of European ancestry. As a consequence, PGx testing panels can inaccurately assign metabolizer phenotypes. For example, the CYP2D6 * 29 decreased function allele is uncommon among individuals of European ancestry (0.1\%, range: $0-2 \%)$ but common among those of African ancestry (9\%, range: 4-20\%) [111]. A PGx panel that did not include this allele would incorrectly assign the *1 or ${ }^{*} 2$ alleles (depending on the other variants being tested). The * 1 allele is a default (not tested) allele that is assigned when none of the tested alleles are detected, while the ${ }^{*} 2$ is a tested allele that has some overlap with the ${ }^{*} 29$ allele. Both the ${ }^{*} 1$ and ${ }^{*} 2$ alleles are interpreted as "normal," and, as such, inadvertent assignment of these alleles could lead to inaccurate metabolizer phenotype predictions (e. g., assigning a person as a NM when they are an IM). Thus, a "normal" genotype result for an individual, particularly those of non-European ancestry, should be interpreted in the context of the alleles that were tested to avoid potential inappropriate medication selection or dosing decisions. Additional information and examples regarding the assignment of alleles can be found in the CYP2C19 [112] and CYP2D6 [74] GeneFocus papers.

\section{Conclusions}

PGx testing should be viewed as a decision-support tool to assist in thoughtful implementation of good clinical care, enhancing rather than offering an alternative to standard treatment protocols. In this context, genetic markers can supplement demographic (e. g., age, sex, family history), clinical (e. g., concomitant medications), and lifestyle (e. g., diet, smoking) information to help guide treatment decisions. At this time, the published evidence, prescribing guidelines, and product labels support use of PGx testing to guide medication selection and dosing in several clinical contexts, particularly for antidepressants (CYP2C19 and CYP2D6), antipsychotics (CYP2D6), anticonvulsants (CYP2C9, HLA-A, and HLA-B), and the $A D H D$ medication atomoxetine (CYP2D6). The current evidence does not support the use of genetic variants in pharmacodynamic genes (e. g., SLC6A4, COMT, MTHFR) to inform prescribing of psychiatric medications. Clinicians and patients are encouraged to edu- 
- Table 4 Estimated phenotype frequency by ancestry for CYP2D6, CYP2C19, CYP2C9, HLA-A and HLA-B.

\begin{tabular}{|c|c|c|c|c|c|c|c|c|c|}
\hline $\begin{array}{l}\text { Genotype-predicted } \\
\text { phenotypes }\end{array}$ & African & $\begin{array}{l}\text { African } \\
\text { American }\end{array}$ & $\begin{array}{l}\text { Caucasian } \\
\text { (European+ } \\
\text { North American) }\end{array}$ & $\begin{array}{l}\text { Near } \\
\text { Eastern }\end{array}$ & $\begin{array}{l}\text { East } \\
\text { Asian }\end{array}$ & $\begin{array}{l}\text { South/ } \\
\text { Central } \\
\text { Asian }\end{array}$ & Americas & Latino & Oceanian \\
\hline \multicolumn{10}{|l|}{ CYP2D6 } \\
\hline Ultrarapid Metabolizer & $4.4 \%$ & $4.5 \%$ & $3.1 \%$ & $9.5 \%$ & $0.7 \%$ & $2.2 \%$ & $5.5 \%$ & $4.4 \%$ & $20.0 \%$ \\
\hline Normal Metabolizer & $43.4 \%$ & $55.7 \%$ & $51.1 \%$ & $54.7 \%$ & $51.9 \%$ & $62.1 \%$ & $63.6 \%$ & $59.2 \%$ & $67.0 \%$ \\
\hline Intermediate Metabolizer & $43.5 \%$ & $36.2 \%$ & $39.0 \%$ & $29.9 \%$ & $39.2 \%$ & $29.5 \%$ & $23.6 \%$ & $29.1 \%$ & $10.1 \%$ \\
\hline Poor Metabolizer & $1.5 \%$ & $2.3 \%$ & $6.5 \%$ & $2.2 \%$ & $0.9 \%$ & $2.3 \%$ & $2.2 \%$ & $3.1 \%$ & $0.4 \%$ \\
\hline \multicolumn{10}{|l|}{ CYP2C19 } \\
\hline Ultrarapid Metabolizer & $3.0 \%$ & $4.3 \%$ & $4.7 \%$ & $3.7 \%$ & $0.0 \%$ & $2.9 \%$ & $0.7 \%$ & $2.8 \%$ & $0.3 \%$ \\
\hline Rapid Metabolizer & $19.0 \%$ & $23.7 \%$ & $27.2 \%$ & $25.7 \%$ & $2.5 \%$ & $18.6 \%$ & $13.6 \%$ & $24.1 \%$ & $2.1 \%$ \\
\hline Normal Metabolizer & $30.1 \%$ & $32.8 \%$ & $39.6 \%$ & $45.2 \%$ & $38.1 \%$ & $29.6 \%$ & $62.8 \%$ & $52.5 \%$ & $3.5 \%$ \\
\hline Intermediate Metabolizer & $36.2 \%$ & $31.4 \%$ & $26.0 \%$ & $23.5 \%$ & $45.9 \%$ & $40.8 \%$ & $21.4 \%$ & $19.0 \%$ & $36.9 \%$ \\
\hline $\begin{array}{l}\text { Likely Intermediate } \\
\text { Metabolizer }\end{array}$ & $4.0 \%$ & $2.8 \%$ & $0.1 \%$ & $0.0 \%$ & $0.1 \%$ & $0.0 \%$ & $0.0 \%$ & $0.4 \%$ & $0.0 \%$ \\
\hline Poor Metabolizer & $6.3 \%$ & $4.1 \%$ & $2.4 \%$ & $1.9 \%$ & $13.0 \%$ & $8.2 \%$ & $1.5 \%$ & $1.1 \%$ & $57.1 \%$ \\
\hline Likely Poor Metabolizer & $1.4 \%$ & $0.7 \%$ & $0.0 \%$ & $0.0 \%$ & $0.0 \%$ & $0.0 \%$ & $0.0 \%$ & $0.0 \%$ & $0.0 \%$ \\
\hline \multicolumn{10}{|l|}{ CYP2C9 } \\
\hline Normal metabolizer & $73.1 \%$ & $75.9 \%$ & $62.9 \%$ & $61.1 \%$ & $83.8 \%$ & $60.0 \%$ & $83.1 \%$ & $74.6 \%$ & $91.2 \%$ \\
\hline Intermediate metabolizer & $26.3 \%$ & $23.6 \%$ & $34.5 \%$ & $36.0 \%$ & $15.2 \%$ & $36.3 \%$ & $16.4 \%$ & $24.5 \%$ & $8.7 \%$ \\
\hline Poor metabolizer & $0.5 \%$ & $0.5 \%$ & $2.6 \%$ & $3.0 \%$ & $0.6 \%$ & $3.8 \%$ & $0.4 \%$ & $1.0 \%$ & $0.1 \%$ \\
\hline \multicolumn{10}{|l|}{ HLA } \\
\hline$A^{*} 31: 01$ & $0.8 \%$ & $1.0 \%$ & $2.6 \%$ & $1.1 \%$ & $3.5 \%$ & $3.3 \%$ & $6.2 \%$ & $4.5 \%$ & $1.1 \%$ \\
\hline$B * 15: 02$ & $0.0 \%$ & $0.1 \%$ & $0.0 \%$ & $0.0 \%$ & $4.6 \%$ & $2.6 \%$ & $0.2 \%$ & $0.0 \%$ & $0.8 \%$ \\
\hline
\end{tabular}

cate themselves or consult an expert prior to ordering a PGx test. This is particularly important given that PGx testing is currently not regulated, and many of the available tests include genes that have little to no support for clinical implementation. Recommendations produced by these tests could lead to inappropriate medication selection and dosing decisions. Various resources to assist in the interpretation and implementation of test results exist, but these resources do not supplant clinical judgement.

A number of larger PGx studies, such as the Ubiquitous Pharmacogenomics Project in Europe [113] and the Precision Medicine in Mental Health Care Study in the United States (NCT03170362) are underway. We expect with the completion of these studies and others that the PGx evidence will continue to evolve, barriers to testing will be cleared, and the uptake of genome sequencing and population-level precision medicine initiatives will increase. As such, we anticipate $\mathrm{PGx}$ testing will become an important tool in psychiatry, mitigating the trial-and-error process that too many individuals currently endure.

\section{Funding}

The work was supported in part by an Alberta Innovates Strategic Research Project G2018000868 (Drs. Aitchison and Bousman), the Neuroscience and Mental Health Institute and Department of Psychiatry, University of Alberta (Ms. Behroozi Asi), NARSAD Young Investigator Grant from the Brain \& Behaviour Research Foundation
(Dr. Amare), the Foundation Bettencourt-Schueller (Dr. Chaumette), the European COST Action EnGagE CA17130 (Dr. Degenhardt), the German Federal Ministry of Education and Research e:Med programme (Dr. Degenhardt), the NIH/NHGRI U24 HG010615 (Drs. Klein and Sangkuhl), the NIH/NIGMS R24 GM123930 (Dr. Gaedigk), the NIDA R01 DA038076 (Dr. Li-Shiun), and the NIMH Intramural Research Program (Dr. McMahon).

\section{Author Contribution}

All authors were involved in the conception of this work. All authors either drafted or critically revised the content and approved the final version. All authors accept accountability for all aspects of the work.

\section{Data Availability}

Data availability is not applicable to this article as no new data were created or analyzed in this study.

\section{Conflict of Interest}

Dr. Bousman reports a grant from Alberta Innovates Strategic Research Project G2018000868, during the conduct of the study; and he has received in-kind testing kits from Myriad Neuroscience, CNSDose, Genomind, and $A B-B i o t i c s$ for research purposes but has not received payments 
or received any equity, stocks, or options in these companies or any other pharmacogenetic companies. Dr. Bengesser has nothing to disclose. Dr. Aitchison reports grants from Alberta Innovates Strategic Research Project G2018000868, other from Neuroscience and Mental Health Institute, other from Department of Psychiatry, during the conduct of the study; non-financial support from HLS Therapeutics, grants from Janssen Inc., Canada, outside the submitted work; and Member, Pharmacogene Variation Consortium (PharmVar); Coauthor, Haplotype Translators for CYP2D6 and CYP2C19; Member, Alberta Cannabis Research and Innovation Network; Member, Schizophrenia Society of Alberta; Board Member, Canadian Consortium for Early Intervention in Psychosis. Dr. Amare has nothing to disclose. Dr. Aschauer has nothing to disclose. Dr. Baune has nothing to disclose. Ms. Behroozi Asl reports grants from Alberta Innovates, during the conduct of the study. Dr. Bishop reports personal fees from OptumRx, outside the submitted work; and he is a member of the Clinical Pharmacogenetics Implementation Consortium. Dr. Burmeister reports personal fees from Chinese University of Hong Kong (Shen Zhen), personal fees from Research Grants Council (RGC) of Hong Kong, personal fees from Alexander von Humboldt Foundation, outside the submitted work. Dr. Chaumette reports personal fees from Janssen-Cilag, grants from Fondation Bettencourt-Schueller, outside the submitted work. Dr. Chen has nothing to disclose. Dr. Cordner has nothing to disclose. Dr. Deckert reports grants from DFG, grants from BMBF, grants from Vogel-Foundation, grants from EU, grants from Bavarian Secretary of Commerce, outside the submitted work. Dr. Degenhardt has nothing to disclose. Dr. DeLisi has nothing to disclose. Dr. Folkersen has nothing to disclose. Dr. Kennedy has a patent 'PGx of Antipsychotic Response'; and 'PGx of Wt Gain' both pending. No licensees. Dr. Kennedy's affiliated hospital, (which is not his employer) the Centre for Addiction and Mental Health, is a part owner of the Canadian subsidiary of Myriad Neuroscience, USA. Dr. Klein reports grants from NIH/NHGRI, during the conduct of the study. Dr. McClay has nothing to disclose. Dr. McMahon has nothing to disclose. Dr. Musil reports personal fees from Otsuka/Lundbeck, outside the submitted work. Dr. Saccone reports that her spouse is listed as an inventor on Issued U.S. Patent 8,080,371, "Markers for Addiction" covering the use of certain single nucleotide polymorphisms in determining the diagnosis, prognosis, and treatment of addiction. Dr. Sangkuhl reports grants from NIH/NHGRI, during the conduct of the study. Dr. Stowe has nothing to disclose. Dr. Tan has nothing to disclose. Dr. Tiwari reports that he is a co-investigator on two pharmacogenetic studies where genetic test kits were provided as in-kind contribution by Assurex Health (Myriad Neuroscience) but he did not receive any payments or any equity, stocks, or options from this company or any other pharmacogenetic companies. Dr. Tiwari is also a co-inventor on a patent assessing risk for antipsychotic-induced weight gain. Dr. C. Zai has a patent for suicide markers issued, and a patent for antipsychotic-induced weight gain markers pending. Dr. G. Zai has nothing to disclose. Dr. Zhang has nothing to disclose. Dr. Gaedigk has nothing to disclose. Dr. Müller reports to be a co-investigator on two pharmacogenetic studies where genetic test kits were provided as in-kind contribution by Myriad Neuroscience. He did not receive any payments or any equity, stocks, or options from any pharmacogenetic companies. Dr. Müller is also a co-inventor on two patent assessing risk for antipsychotic-induced weight gain (pending).

\section{References}

[1] Hiemke C, Bergemann N, Clement HW et al. Consensus guidelines for therapeutic drug monitoring in neuropsychopharmacology: Update 2017. Pharmacopsychiatry 2018; 51: e1

[2] Müller DJ, Rizhanovsky Z. From the origins of pharmacogenetics to first applications in psychiatry. Pharmacopsychiatry 2020; 53 : 155-161

[3] Haga SB, Kantor A. Horizon scan of clinical laboratories offering pharmacogenetic testing. Health Aff (Millwood) 2018; 37: 717-723
[4] Bousman CA, Hopwood M. Commercial pharmacogenetic-based decision-support tools in psychiatry. Lancet Psychiatry 2016; 3: 585-590

[5] Müller D], Kekin I, Kao AC et al. Towards the implementation of CYP2D6 and CYP2C19 genotypes in clinical practice: Update and report from a pharmacogenetic service clinic. Int Rev Psychiatry 2013; 25: 554-571

[6] [Anonymous] Genetic testing and psychiatric disorders: A statement from the International Society of Psychiatric Genetics. Brentwood, TN: International Society of Psychiatric Genetics; 2019

[7] Henriques B, Lapetina D, Yavorskyy V et al. How can drug metabolism and transporter genetics inform psychotropic prescribing? Front Genet in press. doi: 10.3389/fgen.2020.491895

[8] Gaedigk A, Ingelman-Sundberg M, Miller NA et al. The Pharmacogene Variation (PharmVar) Consortium: Incorporation of the Human Cytochrome P450 (CYP) Allele Nomenclature Database. Clin Pharmacol Ther 2018; 103: 399-401

[9] Gaedigk A, Sangkuhl K, Whirl-Carrillo M et al. The evolution of PharmVar. Clin Pharmacol Ther 2019; 105: 29-32

[10] Caudle KE, Dunnenberger HM, Freimuth RR et al. Standardizing terms for clinical pharmacogenetic test results: Consensus terms from the Clinical Pharmacogenetics Implementation Consortium (CPIC). Genet Med 2017; 19: 215-223

[11] Blumenthal D. Pharmacodynamics: Molecular mechanisms of drug action. In: Laurence L. Brunton RH-D, Björn C. Knollmann, Ed Goodman \& Gilman's The Pharmacological Basis of Therapeutics. 13th ed 2018: 31-54

[12] Tangamornsuksan W, Chaiyakunapruk N, Somkrua R et al. Relationship between the HLA-B * 1502 allele and carbamazepine-induced Stevens-Johnson syndrome and toxic epidermal necrolysis: a systematic review and meta-analysis. JAMA Dermatol 2013; 149: 1025-1032

[13] Hicks JK, Bishop JR, Sangkuhl K et al. Clinical Pharmacogenetics Implementation Consortium (CPIC) Guideline for CYP2D6 and CYP2C19 genotypes and dosing of selective serotonin reuptake inhibitors. Clin Pharmacol Ther 2015; 98: 127-134

[14] Jukić MM, Haslemo T, Molden E et al. Impact of CYP2C19 genotype on escitalopram exposure and therapeutic failure: A retrospective study based on 2087 patients. Am J Psychiatry 2018; 175: 463-470

[15] Bråten LS, Haslemo T, Jukic MM et al. Impact of CYP2C19 genotype on sertraline exposure in 1200 Scandinavian patients. Neuropsychopharmacology 2020; 45: 570-576

[16] Sinyor M, Schaffer A, Levitt A. The sequenced treatment alternatives to relieve depression (STAR * D) trial: A review. Can J Psychiatry 2010; 55: $126-135$

[17] Uher R, Perroud N, Ng MY et al. Genome-wide pharmacogenetics of antidepressant response in the GENDEP project. Am J Psychiatry 2010; 167: 555-564

[18] Investigators G, Investigators M, Investigators SD. Common genetic variation and antidepressant efficacy in major depressive disorder: $\mathrm{A}$ meta-analysis of 3 genome-wide pharmacogenetic studies. Am J Psychiatry 2013; 170: 207-217

[19] Probst-Schendzielorz K, Scholl C, Efimkina O et al. CHL1, ITGB3, and SLC6A4 gene expression and antidepressant drug response: results from the Munich Antidepressant Response Signature (MARS) study. Pharmacogenomics 2015; 16: 689-701

[20] Biernacka JM, Sangkuhl K, Jenkins $G$ et al. The International SSRI Pharmacogenomics Consortium (ISPC): A genome-wide association study of antidepressant treatment response. Transl Psychiatry 2015; 5: e553

[21] Whirl-Carrillo M, McDonagh EM, Hebert JM et al. Pharmacogenomics knowledge for personalized medicine. Clin Pharmacol Ther 2012; 92: 414-417 
[22] FDA. The FDA warns against the use of many genetic tests with unapproved claims to predict patient response to specific medications: FDA Safety Communication Available at: https://www.fda.gov/ medical-devices/safety-communications/fda-warns-against-usemany-genetic-tests-unapproved-claims-predict-patient-responsespecific 2019

[23] Hicks JK, Bishop JR, Gammal RS et al. A call for clear and consistent communications regarding the role of pharmacogenetics in antidepressant pharmacotherapy. Clin Pharmacol Ther 2020; 107: 50-52

[24] Hicks JK, Sangkuhl K, Swen J] et al. Clinical pharmacogenetics implementation consortium guideline (CPIC) for CYP2D6 and CYP2C19 genotypes and dosing of tricyclic antidepressants: 2016 update. Clin Pharmacol Ther 2016; 102: 37-44

[25] Swen J], Nijenhuis M, de Boer A et al. Pharmacogenetics: From bench to byte-an update of guidelines. Clin Pharmacol Ther 2011; 89: 662-673

[26] [Anonymous] https://www.knmp.nl/patientenzorg/medicatiebewaking/farmacogenetica/pharmacogenetics-1/pharmacogenetics KNMP Apothekersorganisatie. 2019

[27] Zhang L, Brown S], Shan Y et al. CYP2D6 genetic polymorphisms and risperidone pharmacokinetics: A systematic review and meta-analysis. Pharmacotherapy 2020; 40: 632-647

[28] Jukic MM, Smith RL, Haslemo T et al. Effect of CYP2D6 genotype on exposure and efficacy of risperidone and aripiprazole: A retrospective, cohort study. Lancet. Psychiatry 2019; 6: 418-426

[29] Cui Y, Yan H, Su Y et al. Genotype-based dose recommendations for risperidone in Asian people. Front Pharmacol 2020; 11: 936

[30] Kapur S, Mamo D. Half a century of antipsychotics and still a central role for dopamine D2 receptors. Prog Neuropsychopharmacol Biol Psychiatry 2003; 27: 1081-1090

[31] Lieberman JA. Dopamine partial agonists: A new class of antipsychotic. CNS Drugs 2004; 18: 251-267

[32] Yoshida K, Müller DJ. Pharmacogenetics of antipsychotic drug treatment: update and clinical implications. Mol Neuropsychiatry 2020; 5: 1-26

[33] Olesen OV, Linnet K. Contributions of five human cytochrome P450 isoforms to the $\mathrm{N}$-demethylation of clozapine in vitro at low and high concentrations. J Clin Pharmacol 2001; 41: 823-832

[34] Lesche D, Mostafa S, Everall I et al. Impact of CYP1A2, CYP2C19, and CYP2D6 genotype- and phenoconversion-predicted enzyme activity on clozapine exposure and symptom severity. Pharmacogenomics ] 2020; 20: 192-201

[35] Caudle KE, Rettie AE, Whirl-Carrillo M et al. Clinical pharmacogenetics implementation consortium guidelines for CYP2C9 and HLA-B genotypes and phenytoin dosing. Clin Pharmacol Ther 2014; 96: 542-548

[36] Hou L, Heilbronner U, Degenhardt F et al. Genetic variants associated with response to lithium treatment in bipolar disorder: A genomewide association study. Lancet 2016; 387: 1085-1093

[37] Chen CH, Lee CS, Lee MT et al. Variant GADL1 and response to lithium therapy in bipolar I disorder. N Engl J Med 2014; 370: 119-128

[38] Song J, Bergen SE, Di Florio A et al. Genome-wide association study identifies SESTD1 as a novel risk gene for lithium-responsive bipolar disorder. Mol Psychiatry 2017; 22: 1223

[39] Amare AT, Schubert KO, Hou L et al. Association of polygenic score for schizophrenia and hla antigen and inflammation genes with response to lithium in bipolar affective disorder: A genome-wide association study. JAMA Psychiatry 2018; 75: 65-74

[40] Amare AT, Schubert KO, Hou L et al. Association of polygenic score for major depression with response to lithium in patients with bipolar disorder. Mol Psychiatry in press; doi: 10.1038/s41380-020-0689-5
[41] Manson L, Swen J, Guchelaar H. Diagnostic Test criteria for HLA genotyping to prevent drug hypersensitivity reactions: A systematic review of actionable HLA recommendations in CPIC and DPWG Guidelines. Frontiers in Pharmacology, in press. doi: 10.3389/ fphar.2020.567048

[42] Bloch KM, Sills G], Pirmohamed M et al. Pharmacogenetics of antiepileptic drug-induced hypersensitivity. Pharmacogenomics 2014; 15: 857-868

[43] Deng Y, Li S, Zhang L et al. Association between HLA alleles and lamotrigine-induced cutaneous adverse drug reactions in Asian populations: A meta-analysis. Seizure 2018; 60: 163-171

[44] Phillips EJ, Sukasem C, Whirl-Carrillo M et al. Clinical pharmacogenetics implementation consortium guideline for hla genotype and use of carbamazepine and oxcarbazepine: 2017 update. Clin Pharmacol Ther 2018; 103: 574-581

[45] Satapornpong P, jinda P, Jantararoungtong $T$ et al. Genetic diversity of HLA class I and class II alleles in Thai populations: Contributions to genotype-guided therapeutics. Front Pharmacol 2020; $11: 78$

[46] Lin CW, Huang WI, Chao PH et al. Temporal trends and patterns in carbamazepine use, related severe cutaneous adverse reactions, and HLA-B * 15:02 screening: A nationwide study. Epilepsia 2018; 59: 2325-2339

[47] Chen Z, Liew D, Kwan P. Effects of a HLA-B * 15:02 screening policy on antiepileptic drug use and severe skin reactions. Neurology 2014; 83: 2077-2084

[48] Sukasem C, Chantratita W. A success story in pharmacogenomics: genetic ID card for SJS/TEN. Pharmacogenomics 2016; 17: 455-458

[49] Finsterer J, Segall L. Drugs interfering with mitochondrial disorders. Drug Chem Toxicol 2010; 33: 138-151

[50] Seo T, Nagata R, Ishitsu T et al. Impact of CYP2C19 polymorphisms on the efficacy of clobazam therapy. Pharmacogenomics 2008; 9: 527-537

[51] de Leon J, Spina E, Diaz F]. Clobazam therapeutic drug monitoring: A comprehensive review of the literature with proposals to improve future studies. Ther Drug Monit 2013; 35: 30-47

[52] Sohn DR, Kusaka M, Ishizaki T et al. Incidence of S-mephenytoin hydroxylation deficiency in a Korean population and the interphenotypic differences in diazepam pharmacokinetics. Clin Pharmacol Ther 1992; 52: 160-169

[53] Tomasi J, Lisoway A], Zai CC et al. Towards precision medicine in generalized anxiety disorder: review of genetics and pharmaco(epi) genetics. J Psychiatr Res 2019; 119: 33-47

[54] Brown JT, Bishop JR, Sangkuhl K et al. Clinical pharmacogenetics implementation consortium guideline for cytochrome $\mathrm{P} 450$ (CYP)2D6 genotype and atomoxetine therapy. Clin Pharmacol Ther 2019; 106: 94-102

[55] Stage C, Dalhoff K, Rasmussen HB et al. The impact of human CES1 genetic variation on enzyme activity assessed by ritalinic acid/ methylphenidate ratios. Basic Clin Pharmacol Toxicol 2019; 125: 54-61

[56] Saccone NL, Baurley JW, Bergen AW et al. The value of biosamples in smoking cessation trials: a review of genetic, metabolomic, and epigenetic findings. Nicotine Tob Res 2018; 20: 403-413

[57] Salloum NC, Buchalter ELF, Chanani $S$ et al. From genes to treatments: a systematic review of the pharmacogenetics in smoking cessation. Pharmacogenomics 2018; 19: 861-871

[58] Chen LS, Hartz SM, Baker TB et al. Use of polygenic risk scores of nicotine metabolism in predicting smoking behaviors. Pharmacogenomics 2018; 19: 1383-1394

[59] Lerman C, Schnoll RA, Hawk LW et al. Use of the nicotine metabolite ratio as a genetically informed biomarker of response to nicotine patch or varenicline for smoking cessation: A randomised, doubleblind placebo-controlled trial. Lancet Respir Med 2015; 3: 131-138 
[60] Dennis BB, Bawor M, Thabane L et al. Impact of ABCB1 and CYP2B6 genetic polymorphisms on methadone metabolism, dose and treatment response in patients with opioid addiction: A systematic review and meta-analysis. PLoS One 2014; 9: e86114

[61] Crist RC, Reiner BC, Berrettini WH. A review of opioid addiction genetics. Curr Opin Psychol 2019; 27: 31-35

[62] Liu M, Jiang Y, Wedow R et al. Association studies of up to 1.2 million individuals yield new insights into the genetic etiology of tobacco and alcohol use. Nat Genet 2019; 51: 237-244

[63] Berrettini W. A brief review of the genetics and pharmacogenetics of opioid use disorders. Dialogues Clin Neurosci 2017; 19: 229-236

[64] Schwantes-An TH, Zhang J, Chen LS et al. Association of the OPRM1 Variant rs1799971 (A118G) with non-specific liability to substance dependence in a collaborative de novo meta-analysis of EuropeanAncestry cohorts. Behav Genet 2016; 46: 151-169

[65] Bousman C, Maruf AA, Müller D]. Towards the integration of pharmacogenetics in psychiatry: a minimum, evidence-based genetic testing panel. Curr Opin Psychiatry 2019; 32: 7-15

[66] Chua EW, Kennedy MA. Current state and future prospects of direct-to-consumer pharmacogenetics. Front Pharmacol 2012; 3: 152

[67] Fan M, Bousman CA. Commercial pharmacogenetic tests in psychiatry: do they facilitate the implementation of pharmacogenetic dosing guidelines? Pharmacopsychiatry 2020; 53: 174-178

[68] Bousman CA, Jaksa P, Pantelis C. Systematic evaluation of commercial pharmacogenetic testing in psychiatry: A focus on CYP2D6 and CYP2C19 allele coverage and results reporting. Pharmacogenet Genomics 2017; 27: 387-393

[69] de Leon J, Spina E. What is needed to incorporate clinical pharmacogenetic tests into the practice of psychopharmacotherapy? Expert Rev Clin Pharmacol 2016; 9: 351-354

[70] Pratt VM, Cavallari LH, Del Tredici AL et al. Recommendations for clinical CYP2C9 genotyping allele selection: A joint recommendation of the Association for Molecular Pathology and College of American Pathologists. J Mol Diagn 2019; 21: 746-755

[71] Pratt VM, Del Tredici AL, Hachad $\mathrm{H}$ et al. Recommendations for clinical CYP2C19 genotyping allele selection: A report of the Association for Molecular Pathology. J Mol Diagn 2018; 20: 269-276

[72] Bousman CA, Zierhut H, Müller DJ. Navigating the labyrinth of pharmacogenetic testing: A guide to test selection. Clin Pharmacol Ther 2019; 106: 309-312

[73] Nofziger C, Paulmichl M. Accurately genotyping CYP2D6: not for the faint of heart. Pharmacogenomics 2018; 19: 999-1002

[74] Nofziger C, Turner A], Sangkuhl K et al. PharmVar GeneFocus: CYP2D6. Clin Pharmacol Ther 2020; 107: 154-170

[75] Ingelman-Sundberg M, Mkrtchian S, Zhou $Y$ et al. Integrating rare genetic variants into pharmacogenetic drug response predictions. Hum Genomics 2018; 12: 26

[76] Haga SB, O‘Daniel JM, Tindall GM et al. Survey of US public attitudes toward pharmacogenetic testing. Pharmacogenomics J 2012; 12: 197-204

[77] Walden LM, Brandl E], Tiwari AK et al. Genetic testing for CYP2D6 and CYP2C19 suggests improved outcome for antidepressant and antipsychotic medication. Psychiatry Res 2019; 279: 111-115

[78] Walden LM, Brandl E], Changasi A et al. Physicians' opinions following pharmacogenetic testing for psychotropic medication. Psychiatry Res 2015; 229: 913-918

[79] Stanek EJ, Sanders CL, Taber KA et al. Adoption of pharmacogenomic testing by US physicians: results of a nationwide survey. Clin Pharmacol Ther 2012; 91: 450-458
[80] Brown L, Eum S, Haga SB et al. Clinical utilization of pharmacogenetics in psychiatry - perspectives of pharmacists, genetic counselors, implementation science, clinicians, and industry. Pharmacopsychiatry 2020; 53: 162-173

[81] McKillip RP, Borden BA, Galecki P et al. Patient perceptions of care as influenced by a large institutional pharmacogenomic implementation program. Clin Pharmacol Ther 2017; 102: 106-114

[82] Haga SB. Challenges of development and implementation of point of care pharmacogenetic testing. Expert Rev Mol Diagn 2016; 16: 949-960

[83] Chan J, Soraya GV, Craig L et al. Rapid detection of HLA-B * 57:01-expressing cells using a label-free interdigitated electrode biosensor platform for prevention of abacavir Hypersensitivity in HIV treatment. Sensors (Basel) 2019; 19: 3543

[84] Paterson J. Pharmacogenetic testing a growing area as pilot projects, research get underway. benefitscanada.com. February 1 2018;

[85] Bousman CA, Arandjelovic K, Mancuso SG et al. Pharmacogenetic tests and depressive symptom remission: a meta-analysis of randomized controlled trials. Pharmacogenomics 2019; 20: 37-47

[86] Rosenblat JD, Lee Y, McIntyre RS. The effect of pharmacogenomic testing on response and remission rates in the acute treatment of major depressive disorder: a meta-analysis. J Affect Disord 2018; 241: 484-491

[87] Greden JF, Parikh SV, Rothschild AJ et al. Impact of pharmacogenomics on clinical outcomes in major depressive disorder in the GUIDED trial: a large, patient- and rater-blinded, randomized, controlled study. J Psychiatr Res 2019; 111: 59-67

[88] Perlis RH, Dowd D, Fava M et al. Randomized, controlled, participantand rater-blind trial of pharmacogenomic test-guided treatment versus treatment as usual for major depressive disorder. Depress Anxiety 2020; 37: 834-841

[89] Zeier Z, Carpenter LL, Kalin NH et al. Clinical implementation of pharmacogenetic decision support tools for antidepressant drug prescribing. Am J Psychiatry 2018; 175: 873-886

[90] Benitez J, Cool CL, Scotti D]. Use of combinatorial pharmacogenomic guidance in treating psychiatric disorders. Per Med 2018; 15: 481-494

[91] Perlis RH, Mehta R, Edwards AM et al. Pharmacogenetic testing among patients with mood and anxiety disorders is associated with decreased utilization and cost: a propensity-score matched study. Depress Anxiety 2018; 35: 946-952

[92] Berm E], Looff M, Wilffert B et al. Economic evaluations of pharmacogenetic and pharmacogenomic screening tests: A systematic review. Second update of the literature. PLoS One 2016; 11: e0146262

[93] Brown LC, Lorenz RA, Li J et al. Economic utility: combinatorial pharmacogenomics and medication cost savings for mental health care in a primary care setting. Clin Ther 2017; 39: e591

[94] Chou WH, Yan FX, de Leon J et al. Extension of a pilot study: Impact from the cytochrome P450 2D6 polymorphism on outcome and costs associated with severe mental illness. J Clin Psychopharmacol 2000; 20: 246-251

[95] Maciel A, Cullors A, Lukowiak AA et al. Estimating cost savings of pharmacogenetic testing for depression in real-world clinical settings. Neuropsychiatr Dis Treat 2018; 14: 225-230

[96] Caudle KE, Sangkuhl K, Whirl-Carrillo M et al. Standardizing CYP2D6 genotype to phenotype translation: consensus recommendations from the Clinical Pharmacogenetics Implementation Consortium and Dutch Pharmacogenetics Working Group. Clin Transl Sci 2019; 13: 116-124

[97] Bousman CA, Eyre HA. "Black box" pharmacogenetic decision-support tools in psychiatry. Braz J Psychiatry 2020; 42: 113-115 
[98] Bousman CA, Dunlop BW. Genotype, phenotype, and medication recommendation agreement among commercial pharmacogeneticbased decision support tools. Pharmacogenomics J 2018; 18 : 613-622

[99] Tandy-Connor S, Guiltinan J, Krempely K et al. False-positive results released by direct-to-consumer genetic tests highlight the importance of clinical confirmation testing for appropriate patient care. Genet Med 2018; 20: 1515-1521

[100] Shiroma PR, Geda YE, Mrazek DA. Pharmacogenomic implications of variants of monoaminergic-related genes in geriatric psychiatry. Pharmacogenomics 2010; 11: 1305-1330

[101] Franconi F, Campesi I. Sex impact on biomarkers, pharmacokinetics and pharmacodynamics. Curr Med Chem 2017; 24: 2561-2575

[102] Sugarman EA, Cullors A, Centeno ] et al. Contribution of pharmacogenetic testing to modeled medication change recommendations in a long-term care population with polypharmacy. Drugs Aging 2016; 33: 929-936

[103] Kisor DF, Bodzin AS. Pharmacogenomics in liver transplantation: testing the recipient and the ex-vivo donor liver. Pharmacogenomics 2018; 19: 753-756

[104] Stanke-Labesque F, Gautier-Veyret E, Chhun S et al. Inflammation is a major regulator of drug metabolizing enzymes and transporters: consequences for the personalization of drug treatment. Pharmacol Ther 2020; 215: 107627-107648

[105] Shah RR, Smith RL. Inflammation-induced phenoconversion of polymorphic drug metabolizing enzymes: hypothesis with implications for personalized medicine. Drug Metab Dispos 2015; 43: $400-410$
[106] Smit C, De Hoogd S, Brüggemann RJM et al. Obesity and drug pharmacology: a review of the influence of obesity on pharmacokinetic and pharmacodynamic parameters. Expert Opin Drug Metab Toxicol 2018; 14: 275-285

[107] Klomp SD, Manson ML, Guchelaar HJ et al. Phenoconversion of cytochrome P450 metabolism: a systematic review. J Clin Med 2020; 9: E2890

[108] de Leon J. Personalizing dosing of risperidone, paliperidone and clozapine using therapeutic drug monitoring and pharmacogenetics. Neuropharmacology 2020; 168: 107656

[109] Brown JT, Bishop JR, Schneiderhan ME. Using pharmacogenomics and therapeutic drug monitoring to guide drug selection and dosing in outpatient mental health comprehensive medication management. Ment Health Clin 2020; 10: 254-258

[110] Gaedigk A, Sangkuhl K, Whirl-Carrillo M et al. Prediction of CYP2D6 phenotype from genotype across world populations. Genet Med 2017; 19: 69-76

[111] PharmGKB. PGx gene-specific information tables www.pharmgkb. org/page/pgxGeneRef accessed September 15, 2020

[112] Botton MR, Whirl-Carrillo M, Del Tredici AL et al. PharmVar GeneFocus: CYP2C19. Clin Pharmacol Ther in press. doi: 10.1002/ cpt. 1973

[113] Blagec K, Koopmann R, Crommentuijn-van Rhenen M et al. Implementing pharmacogenomics decision support across seven European countries: The Ubiquitous Pharmacogenomics (U-PGx) project. J Am Med Inform Assoc 2018; 25: 893-898

\section{Notice}

This article was changed on December 11, 2020.

Conflict of Interest:

some details were incorrect and they have been corrected. 